\title{
Imaging features of fungal pneumonia in haematopoietic stem cell transplant patients
}

\author{
Jonathan W. Revels ${ }^{1 A, E, F}$, Shaimaa A. Fadl ${ }^{2 A, E, F}$, Sherry S. Wang ${ }^{3 A, E, F}$, Heta Ladumor ${ }^{4 E, F}$, Haodong Xu ${ }^{5 A, E, F}$, \\ Gregory Kicska ${ }^{5 A, E, F}$
}

'Department of Radiology, University of New Mexico, MSC 10 5530, 1 University of New Mexico, Albuquerque, NM, USA

2Department of Radiology, Virginia Commonwealth University, West Hospital, Richmond, VA, USA

${ }^{3}$ Department of Radiology and Imaging Sciences, University of Utah, Salt Lake City, UT, USA

${ }^{4}$ Weill Cornell Medicine - Qatar, Qatar Foundation - Education City, Doha, Qatar

${ }^{5}$ Department of Radiology, University of Washington Medical Center, Seattle, WA, USA

\section{Abstract}

Patients who have received haematopoietic stem cell transplantation (HSCT) have a high rate of pulmonary complications, and in this immunosuppressed population, fungal pneumonia is of great concern. Fungal pneumonia can have a similar appearance to non-infectious pulmonary processes in HSCT patients, and radiologists should be familiar with the subtle features that may help to differentiate these disease entities. The focus of this article is on the diagnosis of fungal pneumonia in HSCT patients with an emphasis on radiologists' roles in establishing the diagnosis of fungal pneumonia and the guidance of clinical management.

Key words: infection, radiology, CT, fungal, haematopoietic stem cell transplant.

\section{Introduction}

Approximately 21,000 adults in the United States underwent haematopoietic stem cell transplantation (HSCT) in 2018 , with numbers increasing each year [1]. Up to $60 \%$ of HSCT recipients develop life-threatening complications involving the pulmonary system, which are frequently attributable to either infectious (fungal, bacterial, viral) or non-infectious (idiopathic pneumonia syndrome, respiratory distress syndrome, diffuse alveolar haemorrhage, etc.) aetiologies [2,3]. This article focuses on various types of fungal pneumonia as an infectious complication of HSCT to emphasize the role of the radiologist in diagnosing and differentiating fungal pneumonia from its other mimics of bacterial and viral aetiologies, thereby guiding clinical management.
HSCT, also known as bone marrow transplantation, is used to treat multiple cancers including different types of leukaemias, lymphomas, multiple myeloma, as well as some solid tumours [4,5]. Types of HSCT include autologous HSCT (the patient's own stem cells), allogenic (donated by a related or non-related matched or non-matched individual), or syngeneic (donated by the patient's identical twin). The 100-day mortality rate for HSCT can be as high as $40 \%$ in allotransplant HSCT patients, and 20\% in autologous HSCT patients [2]. Pulmonary complications related to HSCT have been reported to occur in up to $60 \%$ of patients [2,3]. Complications related to HSCT can arise during both the pre-engraftment phase (0-30 days after transplant where neutropaenia is profound), early postengraftment (30-100 days, neutropaenia slowly recovering), or late post-engraftment (> 100 days, neutropaenia

Correspondence address:

Jonathan W. Revels, Department of Radiology, University of New Mexico, MSC 105530, 1 University of New Mexico, Albuquerque, NM, 87131, USA, e-mail: Revels.D0@gmail.com

Authors' contribution:

A Study design - B Data collection · C Statistical analysis · D Data interpretation - E Manuscript preparation · F Literature search · G Funds collection 
continues to recover), and these complications range from infectious to non-infectious aetiologies [2].

Fungal pneumonia is not frequently seen amongst the general population, with exceptions of certain endemic regions of the world (for example the Mississippi River Valley, Ohio River Valley, Southwestern United States, etc.) [6]. However, in the immunosuppressed population, fungal pneumonia becomes a greater concern and can reflect opportunistic (Candida, Aspergillus, or Mucormycosis species) or endemic origin (Blastomyces dermatitidis, Coccidiodes immitis, and Histoplasma caspulatum) of infection (Table 1).

Risk factors for fungal pneumonia in HSCT vary depending on the organism causing pneumonia and are summarized in more detail in Table 2. In general, factors contributing to fungal pneumonia include the patient's primary malignancy, exposure, type of transplant, degree of immunosuppression, use of myeloablative regimen, concomitant infection, and occurrence of graft-versushost disease (GVHD) [7,8]. The complex cellular and biochemical pathways that provide resistance to fungal pathogens in immunocompetent individuals can be disrupted for many reasons in HSCT patients. The first line of defence would ordinarily come from the sinopulmonary epithelial cells, which can be damaged by chemotherapy, radiation, and instrumentation (medical support devices), and post-transplant complications such as mucositis or GVHD [8,9]. Additionally, HSCT may have a suppressed immune system related to overall decreased leukocytes (neutropenia, T-cell depletion, etc.) and/or reduced immune response (for example, impaired neutrophil chemotaxis or myeloperoxidase activity) [8]. The latter may explain why some patients who are not neutropaenic still present with fungal pneumonia.

\section{Clinical manifestations}

Patients who have undergone HSCT and are experiencing pulmonary complications may not necessarily pres-

Table 1. Examples of fungal pneumonia infectious organisms

\section{Examples of fungal pneumonia infectious organisms}

\begin{tabular}{|l|l|}
\hline Aspergillus sp. & A. flavus, A. fumigatus, A. lentulus, A. nidulans, A. niger, A. terreus \\
\hline Cryptococcus sp. & C. gatti, C. neoformans \\
\hline Endemic mycoses & Blastomyces dermatitdis, Coccidiodes immitis, Histoplasma caspulatum, Paracoccidioides brasiliensis \\
\hline Fusarium sp. & F. solani complex, F. oxysporum complex \\
\hline $\begin{array}{l}\text { Mucorales sp. (mucormycosis } \\
\text { or zygomycosis) }\end{array}$ & $\begin{array}{l}\text { Mucor spp., Rhizomucor spp., Rhizopus spp., Apophysomyces spp., Cunnighamella bertholletiae, Lichthemiaia } \\
\text { corymbifera }\end{array}$ \\
\hline Paecilomyces spp. & P. varioti, P. lilacinum \\
\hline Secdosporium spp. & S. apiospermum, S. prolificans \\
\hline Scopularopsis spp. & S. brevicaulis \\
\hline
\end{tabular}

Table 2. Risk factors for fungal pneumonia in haematopoietic stem cell transplantation patients

\begin{tabular}{|c|c|}
\hline Risk factor & \\
\hline Host factors & Underlying hematologic malignancy. It is more common with AML and MDS followed by ALL and aplastic anaemia \\
\hline Exposure & Travel, gardening, construction work \\
\hline Laboratory parameters & $\begin{array}{l}\text { Neutropaenia }^{+++} \\
\text {Lymphopaenia }^{++}\end{array}$ \\
\hline Type of transplant & $\begin{array}{l}\text { Allogeneic HSCT patients have a higher prevalence of invasive fungal infections than autologous HSCT patients (5.8-8.1\% } \\
\text { vs. } 1.2 \% \text {, respectively) [11] }\end{array}$ \\
\hline Conditioning regimen & Myeloablative regimen \\
\hline GVHD & Acute GVHD has greater risk of invasive fungal pneumonia than chronic GVHD \\
\hline Immunosuppression & $\begin{array}{l}\text { Myelosuppressive chemotherapy }{ }^{+++} \\
\text {Steroids }>1 \mathrm{mg} / \mathrm{kg}^{+\mathrm{d}^{+++}} \\
\text {Anti-T-cell therapy }\end{array}$ \\
\hline Other infections & Cytomegalovirus (CMV) has been linked \\
\hline
\end{tabular}

AML - acute myelogenous leukaemia, MDS - myelodysplastic syndrome, ALL - acute lymphoblastic leukaemia, SCT - stem cell transplant, HCT - haematopoietic cell transplantation, GVHD - graft-versus-host disease.

${ }^{+}$indicates slightly increased risk of fungal pneumonia in HSCT patients. ${ }^{++}$indicates moderately increased risk of fungal pneumonia in haematopoietic stem cell transplantation (HSCT) patients.

${ }^{+++}$indicates significantly increased risk of fungal pneumonia in HSCT patients. 


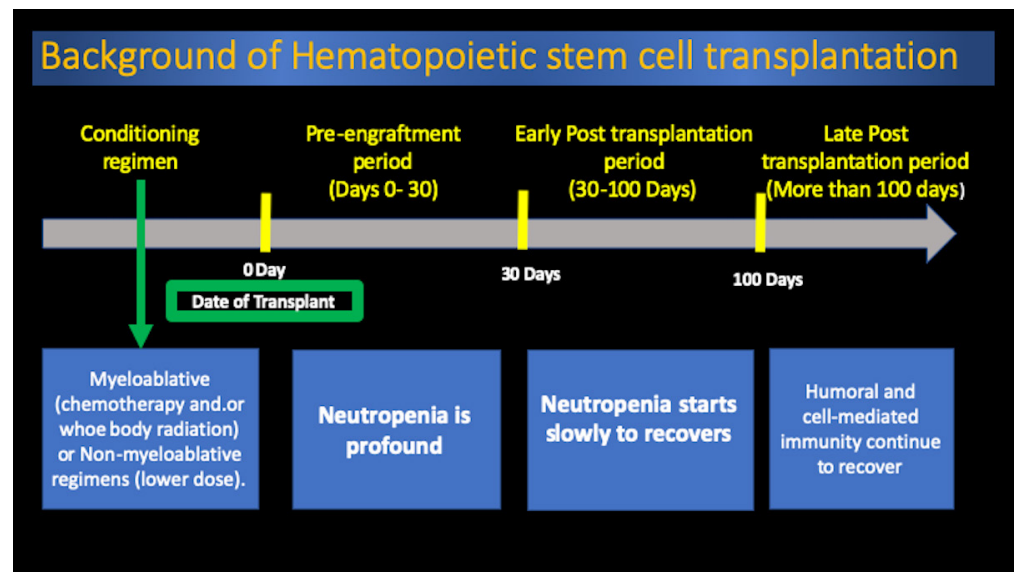

Figure 1. Timeline of infections more commonly encountered after haematopoietic stem cell transplantation and the prophylactic medications generally given to prevent them. Adapted from: Vazquez Guillamet et al. [24]

ent with signs of infection (i.e. fever and leukocytosis) due to immunosuppression. Medical history including an underlying condition requiring HSCT, type of HSCT, elapsed time since HSCT, exposures, and adherence to post-transplant prophylaxis medications can aid in narrowing the broad differential (Figure 1). Persistent fevers, cough, chest pain, and haemoptysis are a few of the clinical features that can be encountered in cases of fungal pneumonia, which may trigger further investigation with radiological studies: chest radiographs and/or computed tomography (CT) [6].

\section{Radiological features of fungal infections}

\section{Opportunistic fungi}

\section{Aspergillus}

Invasive pulmonary aspergillosis (IPA) caused by Aspergillus is the most commonly reported invasive fungal infection seen in upwards of $40 \%$ of HSCT patients [10,11]. Risk factors for invasive Aspergillus pneumonia include allogeneic transplantation, prolonged neutropaenia, CMV infection,
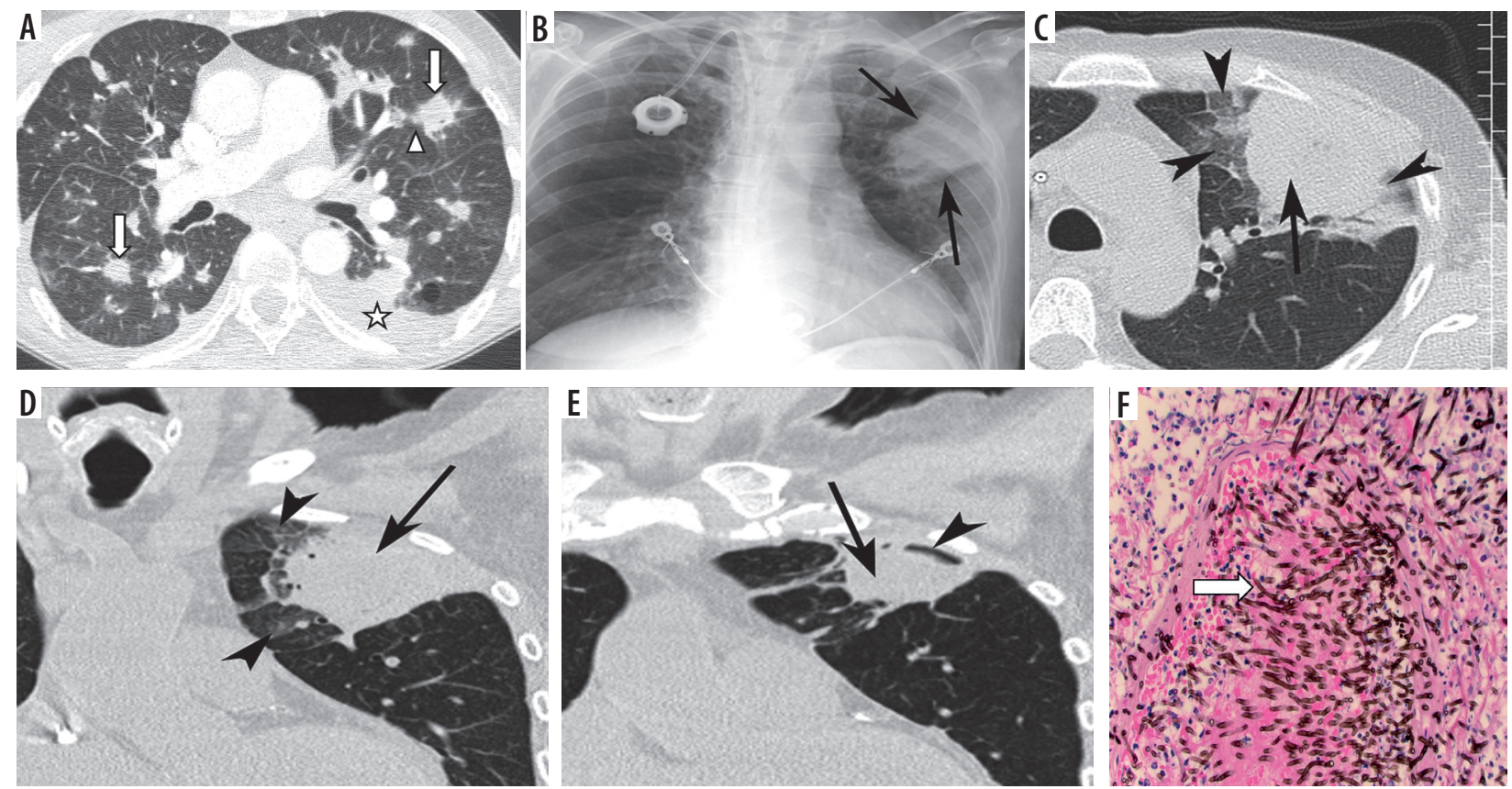

Figure 2. Aspergillus pneumonia. A) 56-year-old patient with acute myelogenous leukaemia undergoing pre-transplant conditioning presented with neutropenic fever diagnosed with Aspergillus nigri pneumonia. Axial chest computed tomography (CT) shows multiple bilateral solid nodules (arrows) with adjacent ground-glass opacities (arrowhead). Small left pleural effusion is also present (star). B-E) Different patient with invasive Aspergillosis pneumonia. A 60-year-old woman with history of acute myelogenous leukaemia status post allogenic stem cell transplant, who presents with persistent fever and pancytopaenia. B) Frontal chest radiograph shows focal peripheral consolidation in the left upper lobe (arrows). (-D) Axial and coronal CT scan show focal consolidation in the left upper lobe (arrow) with adjacent ground glass opacities (arrow heads). E) Coronal CT scan after 3 weeks of antifungal treatment shows decreased size of the focal consolidation (arrow) and the presence of air crescent sign (arrowhead) as part of response to treatment. F) Histopathological microscopy image demonstrating acutely branching, dark, elongated hyphae of Aspergillus 
non-related bone marrow donor, and graft-versus-host disease $[12,13]$. The clinical manifestation of invasive pulmonary aspergillosis usually occurs 2 weeks after the most profound neutropaenia [14]. From a radiologic stance, we will focus on IPA, which has 2 main subtypes: angioinvasive aspergillosis and airway-invasive aspergillosis $[3,6,8]$.

The most common initial manifestations of IPA include solid macronodules ( $\geq 1 \mathrm{~cm}$ in diameter, present in $94 \%$ of patients) with a surrounding halo of ground-glass attenuation classically known as the "halo sign" (present in 61\% of patients) (Figure 2) [6,15-18]. The halo sign is a nonsensitive but highly suggestive finding of early IPA in HSCT recipients $[15,19]$ and represents a distinct nodule with infarction, necrosis, and alveolar haemorrhage [15]. The halo sign is also seen in pneumonia-related to Candida, $\mathrm{Mu}$ cormycosis, herpes simplex virus (HSV), Cytomegalovirus (CMV), and other diseases $[15,18]$. While the halo sign is one of the earliest indicators of IPA, it is also short-lived [20] and can be easily missed if a CT scan is not conducted early during the presentation. Initiation of voriconazole therapy based on the presence of halo sign was associated with a significantly greater response to treatment and survival for IPA [15], which provides support for the usefulness of early CT imaging in suspected IPA.

Other commonly seen findings in IPA include consolidations, cavitary lesions, and air-crescent sign [14,15].
The "hypodense sign" is a highly specific feature of fungal infection [14,21] and represents a relative hypodensity/ attenuation within the pulmonary nodule. The hypodense sign is a precursor to cavitation and the "air crescent sign", which is a late finding specific for IPA, occurring 2-3 weeks after resolution of disease $[6,14]$ and indicating air occupying the space between retracted necrotic lung tissue and the surrounding healthy lung parenchyma. The air crescent sign is seen in recovering IPA and should not be confused with the similar "monod sign", which describes air surrounding an aspergilloma (mycetoma), a focal intracavitary mass in a pre-existing cavity [22].

Less commonly, IPA can affect the airways, referred to as airway invasive aspergillosis, and radiological findings include peribronchial nodular consolidation, branching nodular opacities, centrilobular, and tree-in-bud nodules [23]. Surveillance for aspergillus pneumonia can be performed by measuring aspergillus galactomannan antigen levels (either from bronchoalveolar lavage or serum). However, patients may empirically be given antifungal prophylaxis (such as voriconazole) $[3,9,24]$.

\section{Mucorales}

The diagnosis "mucormycosis" is an umbrella term meaning any infection related to the fungal order of Mucorales,
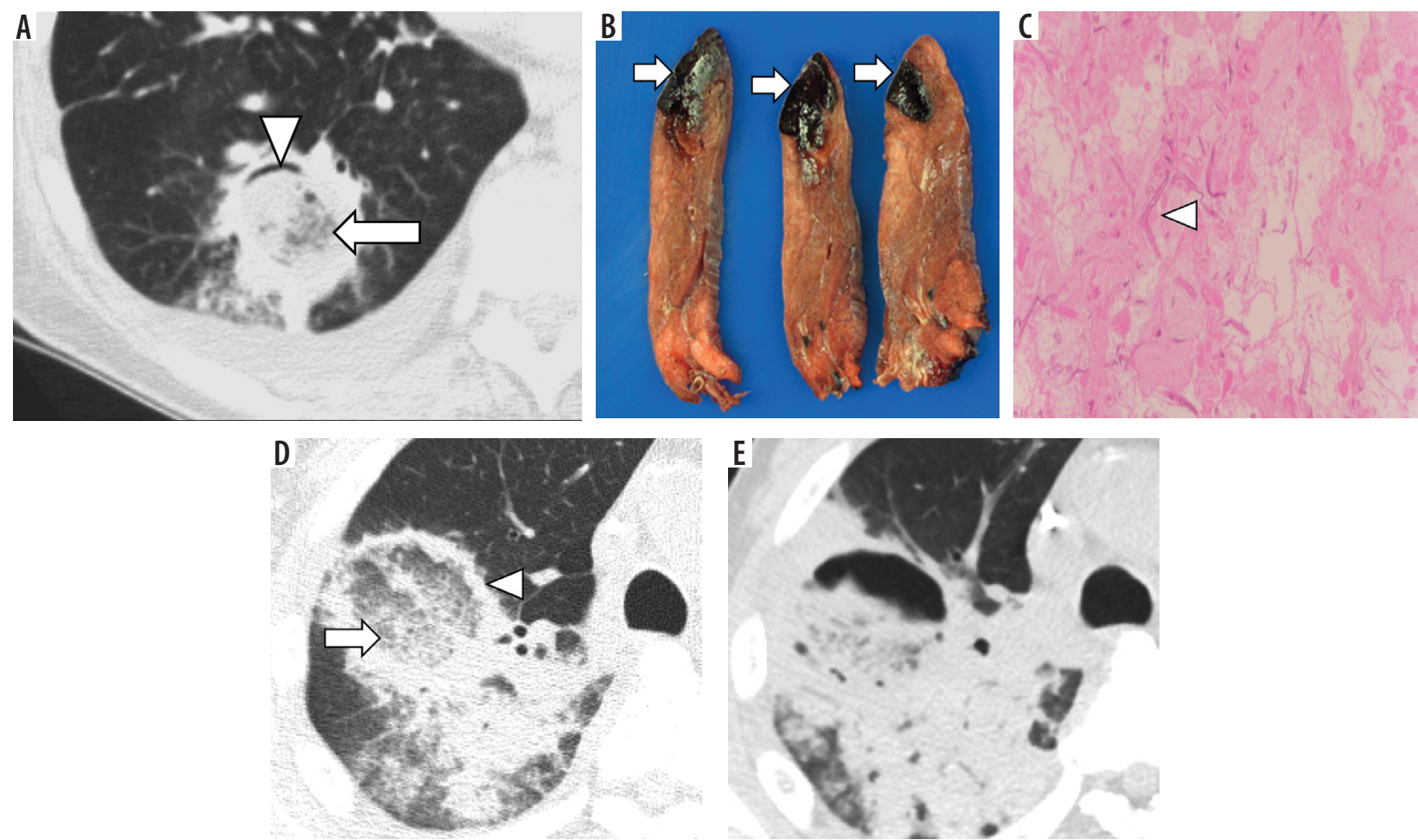

Figure 3. Mucorales pneumonia. A-C) 71-year-old patient with acute myelogenous leukaemia status post haematopoietic stem cell transplantation (HSCT) presented with neutropaenic fever diagnosed with Mucorales pneumonia. A) Axial computed tomography (CT) shows focal consolidation of the right lower lobe with internal ground-glass opacities and peripheral air crescent. B) Gross pathology images after right lobectomy shows focal haemorrhagic infarct in multiple sections (arrows). C) Haematoxylin and eosin histopathology image shows non-septate hyphae with wide angle branching (arrowhead). PCR confirmed Rhizopus microsporus. D-E) Separate patient with history of HSCT. D) Initial axial chest (T shows focal right upper lobe opacity with internal ground-glass (arrow) surrounded by thick nodular rind of consolidation (arrowhead), commonly termed the "reversed halo sign". Final diagnosis was $R h i-$ zomucor meilhi pneumonia. E) Follow-up (T after 1 week shows evolving cavitation and increased adjacent consolidation 
which can be lethal and is often seen in patients with immunocompromising conditions such as HSCT, neutropaenia, diabetic ketoacidosis, or organ transplantation [25]. Rhizopus species account for 70\% of mucormycosis-related fungal pneumonia, with mortality rates $>50 \%$ and preferentially developing in patients with poor phagocytic function [25].

There are some radiological features on CT that can help differentiate mucormycosis from other infections and inflammatory aetiologies, including the collective presence of multiple nodules, pleural effusions, and a "reversed halo" sign (central ground-glass attenuation surrounded by a rind/zone of consolidation) (Figure 3) [19]. Rarely, a filling defect due to pulmonary artery invasion can be seen [23]. However, from an imaging standpoint, the diagnosis of mucormycosis pneumonia may remain indeterminant and serial imaging may be necessary to evaluate changes after initiation of empiric therapy [26]. Treatments for mucormycosis include oral medications (such as isavuconazole), as well as intravenous medications (amphotericin) [24].

\section{Candida}

The prophylactic use of "azole" medications (such as fluconazole) has significantly decreased the rate of Candida species infections (candidiasis) in HSCT patients. The appearance of candidiasis pneumonia overlaps significantly with those discussed previously: nodules, consolidation; possible ground-glass halo; and less common patterns include cavitations and miliary nodules (Figure 4) $[6,27]$.

\section{Cryptococcus}

Cryptococcosis infections are more often associated with conditions related to lymphopaenia and T-cell dysfunction. Cryptococcosis is caused by Cryptococcus neoformans, which can be found in soil throughout the world, more so in areas of bird droppings. The infection can involve multiple organ systems (lungs, skin, central nervous system,

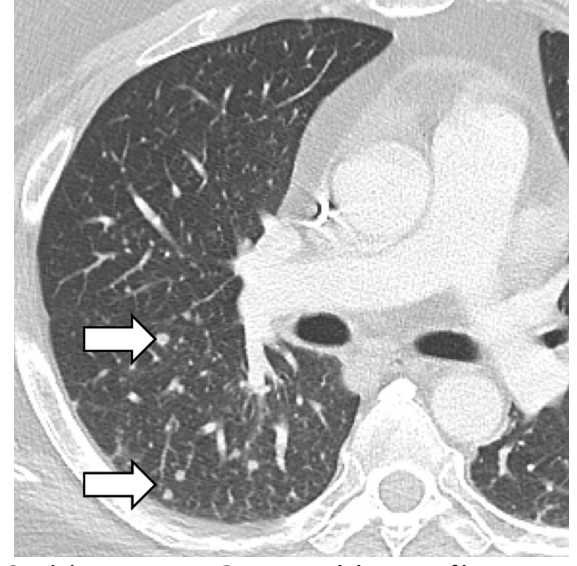

Figure 4. Candida pneumonia. Patient with history of haematopoietic stem cell transplantation. Chest computed tomography demonstrates numerous small pulmonary nodules confirmed to be Candida pneumonia (arrows)

etc.), and patients may present with a wide range of symptoms: fever, mild cough, or acute respiratory failure [28]. Radiological features may be best evaluated on CT and include pulmonary nodules (solitary or multiple in a miliary pattern; possibly cavitary; possibly interstitial with associated reticulations), consolidation, pleural effusion, and lymphadenopathy (Figure 5) [6,28].

\section{Pneumocystis jirovecii}

Pneumocystis jirovecii pneumonia (PJP) has declined in recent years due to the use of routine post-HSCT trimethoprim-sulfamethoxazole (TMP-SMX) prophylaxis [3]. The most common radiological appearance of PJP on CT is upper lobe predominant (although it may also be diffuse), generally peripheral sparing, ground-glass opacities (with or without air-filled cysts), which directly reflects the histopathology of infectious organism causing intra-alveolar accumulation of fibrin and cellular debris [27]. The thinwalled, air-filled cysts can decrease in size and potentially resolve after initiation of treatment, and can also spontane-
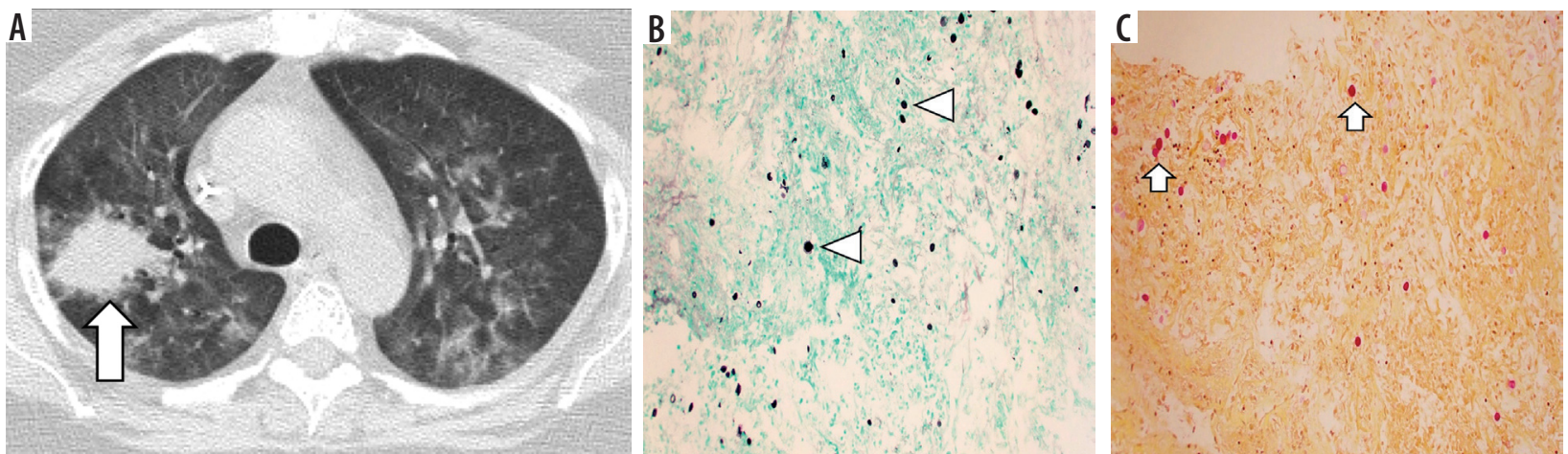

Figure 5. Cryptococcal pneumonia. 61-year-old patient with multiple myeloma status post haematopoietic stem cell transplantation (HSCT) presenting with neutropaenic fever diagnosed with both cryptococcal fungal infection (right lung) and Pneumocystis jirovecii. A) Axial chest computed tomography shows mass-like consolidation in the right upper lobe (arrow), as well as patchy ground-glass opacities. Histopathologic slides: B) Grocott methenamine silver (GMS)-stain showing multiple pleomorphic oval shaped budding (ryptococci (arrow heads). C) Mucarmine stain shows the bright red mucinous capsule which is characteristic of the multiple pleomorphic oval shaped Cryptococci (short arrows) 
ously rupture, resulting in a pneumothorax [28]. Pleural effusion is very uncommon, and the presence of pleural effusion should alert the radiologist to the presence of another pathogen or mixed organisms [23].

\section{Endemic fungi}

Invasive fungal infections due to endemic fungi are very rare and account for $0.6 \%$ of cases among HSCT patients [11], generally occuring 6 months after HSCT transplantation in endemic areas [28].

\section{Histoplasma capsulatum}

In immunocompromised populations, histoplasmosis often appears as disseminated disease with small pulmonary nodules in a military pattern due to hematogenous spread of the fungi $[6,28]$. The presence of pleural effusion and mediastinal involvement is uncommon with histoplasmosis [23]. During the healing process, the pulmonary nodules undergo calcification resulting in a characteristic "target lesion" appearance, which is highly suggestive of a fungal infection of endemic origin $[6,23,28]$.

\section{Coccidioides immitis}

Disseminated coccidioidomycosis radiographically appears very similar to disseminated histoplasmosis with nodules in a military distribution $[23,29]$. A focus of consolidation with hilar and mediastinal adenopathy may also be seen [29].

\section{Blastomyces dermatitidis}

Blastomycosis infections usually present as central focal or patchy consolidation and pulmonary nodules that do not tend to calcify [23].

\section{Differential diagnosis of fungal pneumonia after haematopoietic stem cell transplantation}

Other infections can mimic fungal pneumonia in HSCT patients including bacterial, viral, or less commonly mycobacterial infections. Large nodules and "halo sign" are more suggestive of fungal rather than bacterial or viral aetiologies [30].

Bacterial infections include Gram-negative bacteria, such as Pseudomonas and Escherichia coli in the early phase after HSCT, and Gram-positive bacteria such as Streptococcus and Staphylococcus species in the late phase after HSCT [31]. The most common findings in bacterial infections are areas of consolidation, followed by ground-glass opacities, reticulations, and centrilobular nodules [30]. Often characterized as "fungal-like bacteria", Nocardia and Actinomycosis infections usually appear as nodules or consolidations that cavitate and involve the pleura or chest wall [23].
Viral infections often considered in HSCT patients include CMV pneumonia and RSV pneumonia. The common radiological findings of viral pneumonia are small centrilobular nodules, multifocal consolidations, and ground-glass opacities [30]. In immunocompromised patients, CMV infection can present with patchy areas of consolidations, ground-glass opacities, and small $(<5 \mathrm{~mm})$ nodules [32].

Post-transplant intrathoracic lymphoproliferative disorder (PTLD) should be considered in the differential possibilities when there is a lack of response to antifungals. PTLD has an overall incidence of $1 \%$ in HSCT patients and typically occurs within 6 months of transplant [33]. Pulmonary nodules and masses are the most common radiological finding followed by lymphadenopathy, which appear hypermetabolic on ${ }^{18}$ F-FDG PET/CT [33,34]. False positives due to infectious lesions (such as Aspergillus and Nocardia) $[34,35]$ can be avoided by considering the location of the lesions, the patient's clinical symptoms, and laboratory values such as lactate dehydrogenase levels [35]. PET/CT can help identify other sites of extra-thoracic involvement by the disease and assess treatment response [36]. Pulmonary masses, either solitary or multiple, are the most common thoracic manifestations of PTLD, and a pulmonary mass is seen in $50 \%$ of cases of lung allograft PTLD and nodules in $40-50 \%$ of cases. They can have smooth or irregular borders and homogeneous soft tissue density on CT. Less commonly they can have a hazy halo or central necrosis, which mimics pulmonary aspergillosis [34].

Pulmonary oedema, diffuse alveolar haemorrhage (DAH), idiopathic pneumonia syndrome (IPS) should be considered in patients with diffuse parenchymal opacities and rapidly progressive respiratory symptoms without evidence of infection [33].

Organizing pneumonia is an uncommon late complication of chronic GVHD (2-10\% incidence) [33] and should be considered in patients with consolidations with reverse halo features. Although there is a significant overlap in appearance, organizing pneumonia usually has a smoother rind of peripheral consolidation than in reverse halo sign [33].

\section{Diagnostic approach and management}

Fungal pneumonia can be diagnosed using non-invasive or invasive testing. When fungal pneumonia is suspected, a standard clinical workup can include serum galactomannan (GM), respiratory viral panel, other bloodwork, and imaging. The diagnostic yield of sputum culture is often low [37].

Chest CT and radiography play crucial roles in establishing the likelihood of pneumonia during the infectious workup of HSCT patients. Owing to their wide availability, chest radiographs are often the first-line imaging obtained during an infectious workup in HSCT patients. Radiographs may demonstrate readily apparent features of pneumonia, as well as provide information about the overall extent of disease/ 
severity; however, in cases where the radiographs are normal or when only minimal/subtle features of potential infection are demonstrated, a chest CT should be obtained, given its high negative predictive value in excluding pneumonia (Figure 6) [38]. A normal CT study would suggest an extrapulmonary source of infection.

When pulmonary radiological abnormalities are identified it is important to keep in mind that radiological features of infectious pneumonia can overlap with noninfectious processes, such as pulmonary haemorrhage, organizing pneumonia, and diffuse alveolar damage. A diffuse pattern of disease is most likely to be suggestive of a viral infection and underscores the importance of obtaining a respiratory viral panel in the standard workup. The viral panel should include PCR for influenza virus A or B, respiratory syncytial virus (RSV), parainfluenza viruses, Coronavirus, Rhinovirus, Metapneumovirus, Adenovirus, or Bocavirus [37]. If the viral panel is negative for infection but suspicion of infection persists, bronchoscopy with bronchoalveolar lavage (BAL) is the best next step because the BAL fluid is more sensitive for measuring galactomannan [39] and isolating organisms on Gram stain and culture. If bronchoscopy does not yield a diagnosis but suspicion of infection remains, image-guided percutaneous FNA or biopsy should be considered for definitive diagnosis [8]. Imaging is uniquely useful in this setting to obtain a targeted transbronchial biopsy that has a high diagnostic yield in lesions $>1 \mathrm{~cm}$ [8]. Surgical lung biopsy is often not possible in HSCT recipients due to neutropaenia and thrombocytopaenia [8].

The main goal of imaging in fungal pneumonia is to identify a source of infection before resorting to an invasive procedure, such as bronchoscopy or percutaneous tissue sampling. Additionally, imaging examinations are able to evaluate coexisting abnormalities hindering recovery, as well as alternative diagnoses. Imaging should not be used to suggest a single/specific organism. Ultimately, radiological findings should match the patients' clinical presentation, verified through active communication with the clinical team; the patient's HSCT infectious history is imperative. In an asymptomatic immunocompromised patient, a radiological examination may be the best test to monitor for developing infection, especially because the rate of infection is low but is associated with high mortality [37]. When there is radiological evidence of pneumonia, the role of radiologists is 2-fold: identifying the site of greatest disease burden that can be targeted by endoscopists during BAL, which is becoming standard practice for diagnosis pulmonary infection in HSCT patients [40], as well as suggesting potential infectious aetiologies that could aid in selection of initial medication therapy in cases where endoscopy is not readily available.

Fluconazole is the standard prophylaxis for fungal pneumonia. Triazoles, including voriconazole, are usually used empirically in patients with suspected fungal infections with non-Mucorales spp. Amphotericin, which is considered a relatively toxic antifungal, is used for the treatment of Mucorales spp. Surgical resection is used to treat Mucorales spp.

Radiological follow-up during and after initiation of therapy is usually necessary to evaluate treatment response [37]. Imaging surveillance for response to treatment of infectious pneumonia in HSCT is generally performed with repeat chest radiographs during hospitalization, although chest CT may be utilized as well. The goal of imaging is to document clearance and/or resolution of the infection. When patients are not responding to treatment clinically (persistent infectious symptoms) and/or their pneumonia is worsening radiologically, this typically triggers clinical re-investigation that may include repeat BAL due to possible treatment failure versus development of a new infection [41]. If the patient is clinically responding well and laboratory findings support a positive response, the radiologic features may be merely reflecting a lag time or "pseudo-worsening" that requires longer surveillance to demonstrate a clearance of infection [20]. There are no current guidelines discussing the duration of imaging follow-up for the pulmonary findings in patients who are
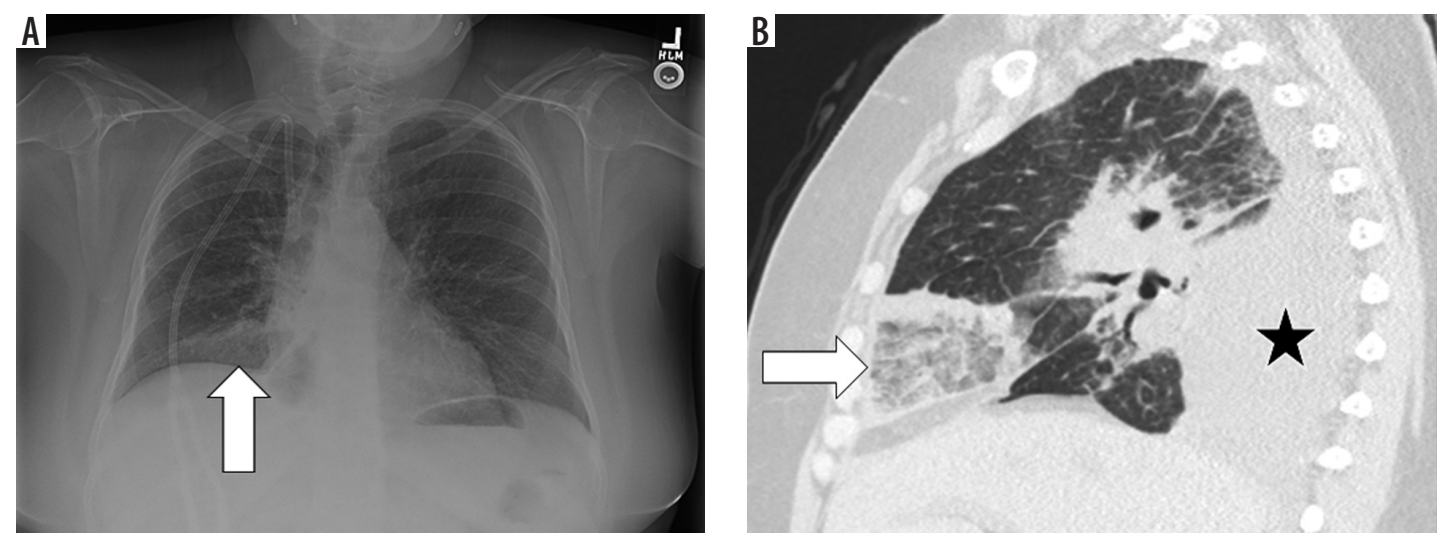

Figure 6. Improved radiologic visualization of Mucorales pneumonia between chest radiograph and computed tomography. A 60-year-old woman with history of stem cell transplant who presents with fever. A) Frontal chest radiograph demonstrates a subtle opacity (arrow) adjacent to the right heart border. B) Sagittal reformat from computed tomography shows central ground-glass opacity (arrowhead) in the right middle lobe surrounded by a rind of consolidation (arrow). There is also associated pleural effusion (star) 
responding positively to treatment, but suffice it to say, close imaging surveillance is very likely warranted, similar to that performed in the general population.

\section{Conclusions}

With an ever-growing population of HSCT patients, radiologists should be familiar with imaging features of fungal pneumonia to raise the possibility of its presence and launch an appropriate clinical investigation, if not currently underway. The imaging appearance of a successfully treated fungal pneumonia includes cavitation, which should not be confused with increased infection. While there is overlap and non-specificity in imaging findings of fungal pneumonia, the presence of certain findings (halo sign or air crescent sign, reverse halo, and target lesion) in conjunction with a relevant medical history and geographical location can help narrow an otherwise broad differential.

\section{Conflict of interest}

The authors report no conflict of interest.

\section{References}

1. D'Souza A, Fretham C, Lee SJ, et al. Current use of and trends in hematopoietic cell transplantation in the United States. Biol Blood Marrow Transplant 2020; 26: e177-e182.

2. Afessa B, Peters SG. Major complications following hematopoietic stem cell transplantation. Semin Respir Crit Care Med 2006; 27: 297-309.

3. Wieruszewski PM, Herasevich S, Gajic O, Yadav H. Respiratory failure in the hematopoietic stem cell transplant recipient. World J Crit Care Med 2018; 7: 62-72.

4. Majhail NS, Farnia SH, Carpenter PA, et al. Indications for autologous and allogeneic hematopoietic cell transplantation: guidelines from the American Society for Blood and Marrow Transplantation. Biol Blood Marrow Transplant 2015; 21: 1863-1869.

5. Ljungman P, Urbano-Ispizua A, Cavazzana-Calvo M, et al. Allogeneic and autologous transplantation for haematological diseases, solid tumours and immune disorders: definitions and current practice in Europe. Bone Marrow Transplant 2006; 37: 439-449.

6. Hussien A, Lin CT. CT findings of fungal pneumonia with emphasis on aspergillosis. Emerg Radiol 2018; 25: 685-689.

7. Pergam SA. Fungal pneumonia in patients with hematologic malignancies and hematopoietic cell transplantation. Clin Chest Med 2017; 38: 279-294.

8. Young AY, Leiva Juarez MM, Evans SE. Fungal pneumonia in patients with hematologic malignancy and hematopoietic stem cell transplantation. Clin Chest Med 2017; 38: 479-491.

9. Nathan S, Ustun C. Complications of stem cell transplantation that affect infections in stem cell transplant recipients, with analogies to patients with hematologic malignancies. Infect Dis Clin North Am 2019; 33: 331-359.

10. Neofytos D, Horn D, Anaissie E, et al. Epidemiology and outcome of invasive fungal infection in adult hematopoietic stem cell transplant recipients: analysis of Multicenter Prospective Antifungal Therapy (PATH) Alliance registry. Clin Infect Dis 2009; 48: 265-273.

11. Kontoyiannis DP, Marr KA, Park BJ, et al. Prospective surveillance for invasive fungal infections in hematopoietic stem cell transplant recipients, 2001-2006: overview of the Transplant-Associated Infection Surveillance Network (TRANSNET) Database. Clin Infect Dis 2010; 50: 1091-1100.

12. Salman N, Törün SH, Budan B, Somer A. Invasive aspergillosis in hematopoietic stem cell and solid organ transplantation. Expert Rev Anti Infect Ther 2011; 9: 307-315.
13. Smith JA, Kauffman CA. Pulmonary fungal infections. Respirology 2012; 17: 913-926.

14. Prasad A, et al. Pulmonary aspergillosis: what CT can offer before it is too late! J Clin Diagn Res 2016; 10: TE01-TE5.

15. Greene RE, Schlamm HT, Oestmann JW, et al. Imaging findings in acute invasive pulmonary aspergillosis: clinical significance of the halo sign. Clin Infect Dis 2007; 44: 373-379.

16. Georgiadou SP, Sipsas NV, Marom EM, Kontoyiannis DP. The diagnostic value of halo and reversed halo signs for invasive mold infections in compromised hosts. Clin Infect Dis 2011; 52: 1144-1155.

17. Alves GR, Marchiori E, Irion K, et al. The halo sign: HRCT findings in 85 patients. J Bras Pneumol 2016; 42: 435-439.

18. Won HJ, Lee KS, Cheon JE, et al. Invasive pulmonary aspergillosis: prediction at thin-section CT in patients with neutropenia - a prospective study. Radiology 1998; 208: 777-782.

19. Marchiori E, Marom EM, Zanetti G, et al. Reversed halo sign in invasive fungal infections: criteria for differentiation from organizing pneumonia. Chest 2012; 142: 1469-1473.

20. Caillot D, Couaillier JF, Bernard A, et al. Increasing volume and changing characteristics of invasive pulmonary aspergillosis on sequential thoracic computed tomography scans in patients with neutropenia. J Clin Oncol 2001; 19: 253-259.

21. Horger M, Einsele H, Schumacher U, et al. Invasive pulmonary aspergillosis: frequency and meaning of the "hypodense sign" on unenhanced CT. Br J Radiol 2005; 78: 697-703.

22. Thompson BH, Stanford W, Galvin JR, Kurihara Y. Varied radiologic appearances of pulmonary aspergillosis. Radiographics 1995; 15: 1273-1284.

23. Orlowski HLP, McWilliams S, Mellnick VM, et al. Imaging spectrum of invasive fungal and fungal-like infections. Radiographics 2017; 37: 1119-1134.

24. Vazquez Guillamet C, Hsu JL, Dhillon G, Vazquez Guillamet R. Pulmonary infections in immunocompromised hosts: clinical. J Thorac Imaging 2018; 33: 295-305.

25. Ibrahim AS, Spellberg B, Walsh TJ, Kontoyiannis DP. Pathogenesis of mucormycosis. Clin Infect Dis 2012; 54 Suppl 1 (Suppl 1): S16-S22.

26. Nam BD, Kim TJ, Lee KS, et al. Pulmonary mucormycosis: serial morphologic changes on computed tomography correlate with clinical and pathologic findings. Eur Radiol 2018; 28: 788-795. 
27. Torres PPTES, Rabahi MF, Moreira MAC, et al. Tomographic assessment of thoracic fungal diseases: a pattern and signs approach. Radiol Bras 2018; 51: 313-321.

28. Chong S, Lee KS, Yi CA, et al. Pulmonary fungal infection: imaging findings in immunocompetent and immunocompromised patients. Eur J Radiol 2006; 59: 371-383.

29. Jude CM, Nayak NB, Patel MK, et al. Pulmonary coccidioidomycosis: pictorial review of chest radiographic and CT findings. Radiographics 2014; 34: 912-925.

30. Escuissato DL, Gasparetto EL, Marchiori E, et al. Pulmonary infections after bone marrow transplantation: high-resolution CT findings in 111 patients. Am J Roentgenol 2005; 185: 608-615.

31. Lossos IS, Breuer R, Or R, et al., Bacterial pneumonia in recipients of bone marrow transplantation. A five-year prospective study. Transplantation 1995; 60: 672-678.

32. Pandey T, Maximin S, Bhargava P. Imaging of complications from hematopoietic stem cell transplant. Indian J Radiol Imaging 2014; 24: 327-338.

33. Pena E, Souza CA, Escuissato DL, et al., Noninfectious pulmonary complications after hematopoietic stem cell transplantation: practical approach to imaging diagnosis. Radiographics 2014; 34: 663-683.

34. Borhani AA, Hosseinzadeh $\mathrm{K}$, Almusa O, et al. Imaging of posttransplantation lymphoproliferative disorder after solid organ transplantation. Radiographics 2009; 29: 981-1000; discussion 1000 1002.

35. Montes de Jesus FM, Kwee TC, Kahle XU, et al. Diagnostic performance of FDG-PET/CT of post-transplant lymphoproliferative disorder and factors affecting diagnostic yield. Eur J Nucl Med Mol Imaging 2020; 47: 529-536.

36. Levine DS, Navarro OM, Chaudry G, et al. Imaging the complications of bone marrow transplantation in children. Radiographics 2007; 27: 307-324.

37. Cheng GS, Stednick ZJ, Madtes DK, et al. Decline in the use of surgical biopsy for diagnosis of pulmonary disease in hematopoietic cell transplantation recipients in an era of improved diagnostics and empirical therapy. Biol Blood Marrow Transplant 2016; 22: $2243-$ 2249.

38. Heussel CP, Kauczor HU, Heussel GE, et al. Pneumonia in febrile neutropenic patients and in bone marrow and blood stem-cell transplant recipients: use of high-resolution computed tomography. J Clin Oncol 1999; 17: 796-805.

39. Wheat LJ, Walsh TJ. Diagnosis of invasive aspergillosis by galactomannan antigenemia detection using an enzyme immunoassay. Eur J Clin Microbiol Infect Dis 2008; 27: 245-251.

40. Chellapandian D, Lehrnbecher T, Phillips B, et al. Bronchoalveolar lavage and lung biopsy in patients with cancer and hematopoietic stem-cell transplantation recipients: a systematic review and metaanalysis. J Clin Oncol 2015; 33: 501-509.

41. Cordonnier C. Pneumonia after hematopoietic stem cell transplantation. In: Ljungman P, Snydman D, Boeckh M (eds.). Transplant Infections. $4^{\text {th }}$ ed. Springer International Publishing, 2016; 251-269. 